\title{
Application of micro-dried droplets for quantitative analysis of particulate inorganic samples with LA-ICP-MS demonstrated on surface-modified nanoparticle $\mathrm{TiO}_{2}$ catalyst materials
}

\author{
Felix Horak $^{1}$ (D) $\cdot$ Andreas Nagl $^{2} \cdot$ Karin Föttinger $^{2} \cdot$ Andreas Limbeck $^{1}$ \\ Received: 6 July 2020 / Accepted: 20 October 2020 / Published online: 5 November 2020 \\ (C) The Author(s) 2020
}

\begin{abstract}
A quick, flexible and reliable method was developed, based on laser ablation inductively coupled plasma mass spectrometry (LAICP-MS), for accurate assessment of nanomaterial composition with sample amounts in the picogram to nanogram range. We demonstrate its capabilities for the analysis of surface-modified $\mathrm{TiO}_{2}$ nanoparticulate (NP) catalyst materials. For sampling, suspensions of NP were deposited on a substrate material, ablated with a pulsed laser and then analysed using quadrupole ICPMS. The calibration and quantification approach is based on the use of so-called micro-dried droplets $(\mu \mathrm{DD})$ as the standard material. To overcome some of the major drawbacks of conventional dried droplet approaches, self-aliquoting wells were used in this work. By mimicking the ablation conditions for the sample and standard, it was possible to create a pseudo-matrix-matched calibration, not only for this specific NP composition but also for a larger variety of samples. A commercially available reference material (AUROlite ${ }^{\mathrm{TM}}$, Strem Chemicals) was used to compare the method against established methods such as slurry analysis and microwave-assisted digestion in combination with subsequent liquid sample measurement. The results obtained with the proposed procedure $(0.74 \% \mathrm{wt} \pm 0.13 \% \mathrm{wt})$ are in good agreement to a certified value $(0.8 \% \mathrm{wt})$ and added an additional layer of information. Due to the significantly reduced sampling size in comparison with the investigated liquid measurement approaches, it was possible to obtain information about the homogeneity of the catalyst material. The results indicate that the AUROlite ${ }^{\mathrm{TM}}$ reference material has a heterogeneous loading which requires more than $300 \mathrm{pg}$ of material to be used to cancel out. This was not observed for the custom materials discussed in this work.
\end{abstract}

Keywords Laser ablation-ICP-MS $\cdot$ Quantitative calibration $\cdot$ Catalyst materials $\cdot \mathrm{TiO}_{2} \cdot$ Surface-modified nanoparticles

\section{Introduction}

Titania is one of the most studied materials for photocatalysis [1] and also of high importance for supported metal nanoparticles in heterogeneous catalysts [2] due to its strong metalsupported interaction with noble metals. Ever since Haruta

\section{Supplementary Information The online version contains} supplementary material available at https://doi.org/10.1007/s00604-02004609-9.

Andreas Limbeck

andreas.limbeck@tuwien.ac.at

1 Institute of Chemical Technologies and Analytics, TU Wien, Getreidemarkt 9/164-IAC, 1060 Vienna, Austria

2 Institute of Materials Chemistry, TU Wien, Getreidemarkt 9/165, 1060 Vienna, Austria et al. [3, 4] reported the, rather surprising, high activity of gold nanoparticles in $\mathrm{CO}$ oxidation, gold received much attention as a catalyst material for a wide range of reactions in both liquid and gas phases, often with titania as the support material. The exact knowledge of the metal loading is crucial to assess the performance of a specific catalyst composition. Determining the number of active sites and thus the calculation of turnover frequencies (TOFs), a key figure in catalysis, relies on this knowledge. Thus, analytical methods for fast and reliable analysis are needed to improve material properties and to support the development of novel compositions.

The characterization of nanoparticle materials is usually performed with so-called bulk analysis methods such as Xray fluorescence analysis with wavelength-dispersive or energy-dispersive (WD/ED) detection XRF or conventional liquid analysis as slurry [5-7] or after digestion [8,9] using atomic absorption spectroscopy (AAS), inductively coupled 
plasma optical emission spectrometry (ICP-OES), or ICP-MS. These methods provide accurate and precise information about the average composition of the analysed material; however, information about the homogeneity of NP materials remains inaccessible with these established methods. Some information about the homogeneity of the individual composition can be derived via SEM/TEM-EDX analysis, but this requires specialized sample preparation and is tedious to evaluate. Analysis of NP agglomerates is also possible using secondary ion mass spectrometry (SIMS), which provides excellent sensitivity and allows investigations with a spatial resolution in the lower submicrometer range; however, quantitative analysis is difficult since SIMS is known to be affected by strong matrix effects [10].

In recent years, laser ablation inductively coupled plasma mass spectrometry (LA-ICP-MS) has proven to be a potent technique for the elemental analysis of small quantities such as single-micron-sized and smaller particles $[11,12]$ and similar non-bulk-like samples such as nanoparticulate suspensions [13, 14]. However, LA-ICP-MS measurements typically struggle with quantification and are therefore most commonly performed semi-quantitatively as an applicable reference material is barely available, especially for novel materials such as surface-modified nanoparticulates. Since the preparation and characterization of in-house standards is laborious and often challenging, an alternative strategy for the production of suitable standard materials is needed.

For this purpose, various approaches based on dried droplets [15-17] have been suggested in the past. Whilst side effects such as the "coffee-stain" effect and size discrimination $[18,19]$ due to increased evaporation rate towards the rim of the droplet could be overcome by complete consumption of the droplet and by addition of an internal standard [20], this procedure can be very time consuming and does not reflect the short transient nature of a particle ablation. Calibration procedures based on modified inkjet printers [21] are also reportedly capable of producing reliable calibration curves for small amounts of samples, though the process of spiking and mixing a specific target element composition can be tedious. To overcome the drawbacks associated with the use of dried droplets, recently, the use of micro-dried droplets ( $\mu \mathrm{DD})$ with diameters smaller than the applied laser beam has been introduced [22]. The use of micro-grooves represented an improvement of this approach as shown by Nischkauer for whole blood analysis [23] or Weiss et al. for the analysis of boride thin films [24]. The fundamental concept of the method is to provide a hydrophilic cavity on an else hydrophobic surface which is then filled with a specific amount of liquid reference solution. As the hydrophobic surface of the substrate is not likely to accumulate any residue from the liquid standard solution, the total amount of deposited material is governed by the dimensions of the hydrophilic wells.
Here, we present a further optimized variant of the $\mu \mathrm{DD}$ approach, which is not limited to the determination of elemental ratios, such as presented in earlier works, but also enables complete quantitative analysis for a large variety of elements. The optimized procedure was applied for the quantitative analysis of surface-modified catalyst materials, both commercially available (AUROlite ${ }^{\mathrm{TM}}$ ) and with a custom-designed composition. To assure the accuracy of the method, it was compared to established conventional elemental analysis techniques such as slurry-ICP-OES and liquid digestion followed by ICP-OES analysis of the investigated certified reference material.

\section{Experimental}

\section{Chemicals, reagents and materials}

p.a. grade ethanol, hydrochloric acid, hydrofluoric acid, hydrogen peroxide solution and nitric acid were purchased from Merck (Germany, https://www.merckmillipore.com) and diluted with purified water prepared by a Barnstead EASYPURE II system (Thermo Fisher Scientific, USA, https://www.fishersci.de) as required. Single-element standards were purchased from Merck ( $\mathrm{Si}, \mathrm{Al}$ and $\mathrm{Gd}), \mathrm{SPEX}$ (https://www.spexcertiprep.com) (U), VWR (https://www. vwr.com) ( $\mathrm{Sr}$ and $\mathrm{Au}$ ), ROTH (https://www.carlroth.com) (Ti) and Alfa Aesar (https://www.alfa.com) (Os). Liquid standard solutions with varying compositions and concentrations were prepared just before use by dilution of the respective single-element standards.

AUROlite $^{\mathrm{TM}}$ (Strem Chemicals, https://www.strem.com) was used as a certified reference composition. For the synthesis of the custom catalyst material, rutile (TP Hombikat Mikrorutil, Venator, https://www.americanelements.com), anatase (Sigma-Aldrich, https://www.sigmaaldrich.com), gold(III) chloride (Sigma-Aldrich) and urea (Merck) were used. Makrolon ${ }^{\circledR}$ (Bayer Material Science, https://www.covestro. com) was selected as the substrate material for the $\mu \mathrm{DD}$ production and the analysis of all solid samples.

\section{Instrumentation}

A NWR213 laser ablation system (esi, http://www.nwrlasers. com) was used for both the preparation of the $\mu \mathrm{DD}$ wells and the respective analysis of particles and reference materials. For the LA-ICP-MS analysis, the NWR213 laser was coupled to a quadrupole ICP-MS (Thermo iCAP Qc, Thermo Fisher Scientific). The transfer line was modified to increase the washout time to allow more observations per analysis run. Determination of the depth and dimensions of individual wells was carried out using a DektakXT profilometer (BRUKER, https://www.bruker.com). 
Samples were ultrasonicated with an ultrasonic cleaner USC 200 TH (VWR) and vortexed with a Vortex Genie 2 (Scientific Industries, https://www.scientificindustries.com).

Sample loading steps as well as loading of the $\mu$ DD cavities were performed in a VFT 1525 ultraclean laminar flow hood (WEISS Technik, https://www.weiss-technik.com).

Microwave-assisted sample digestions were performed using an Anton Paar Multiwave 3000 (Anton Paar, https:// www.anton-paar.com); liquid sample solutions were analysed using an iCAP 6500 ICP-OES spectrometer (Thermo Fisher Scientific) equipped with a CETAC ASX520 autosampler (CETAC Technologies, http://www. teledynecetac.com).

A FEI Quanta 250 FEGSEM (https://www.fei.com) was used for the SEM measurements.

(S)TEM imaging was performed with a FEI Tecnai F20 FEG-TEM instrument.

\section{Synthesis and characterization of custom-modified catalyst particles}

Preparation of the Au nanoparticle catalysts supported on $\mathrm{TiO}_{2}$ was done following a well-established recipe by Zanella et al. [25, 26], which is known to lead to small welldispersed particles (synthesis recipe is provided in ESM). Three different $\mathrm{TiO}_{2}$ supports were employed: rutile, anatase and a physical mixture of $20 \%$ rutile and $80 \%$ anatase. The individual particles tend to agglomerate into larger particles even in the highly diluted solutions used for the SEM and TEM images, as can be observed in Fig. 1, which shows various electron microscopic images of the larger titania NP (Fig. 1a and b) and the smaller Au catalyst NP on top (Fig. 1c). For the SEM measurement, a Schottky emitter with an acceleration voltage of $5.00 \mathrm{kV}$ was applied. The working distance (WD) for the measurements was $5.7 \mathrm{~nm}$ and an EverhartThornley detector (ETD) was used. Due to the limited resolution of the SEM instrument, individual titania NP cannot be resolved and only larger clusters can be observed, and this is even more true for the smaller Au NP. (S)TEM imaging allows analysis of individual particles. The images were made with an acceleration voltage of $200 \mathrm{kV}$. The Au NP which forms during the pretreatment can be observed as bright spots in the TEM image (Fig. 1c). The average size of the deposited $\mathrm{Au}$ NP was determined to be $3.3 \pm 1 \mathrm{~nm}$ by STEM-HAADF with a sample size of $n=292$ particles.

\section{Production of $\mu \mathrm{DD}$ (theory and instrumentation)}

Using a NWR213 ns laser, multiple sets of 100 self-aliquoting wells with a diameter of $50 \mu \mathrm{m}$ were shot in 2-mm-thick PC (Makrolon®) plates. This substrate provides a sufficiently hydrophobic surface and is commercially available with high purity. The length and width of the substrate were freely adjusted to the available space in the ablation cell in the laser. For ease of handling, the slides used in this work were typically cut to match a typical microscope slide $(75 \mathrm{~mm} \times$ $26 \mathrm{~mm}$ ). The typical layout of a $\mu \mathrm{DD}$ array consists of 20 lines with 5 wells resulting in a total amount of 100 wells per array. Within such an array $300 \mu \mathrm{m}$ of distance was left between wells and $5 \mathrm{~mm}$ between arrays of varying concentrations. The wells were shot with a fluence of $1 \mathrm{~J} / \mathrm{cm}^{2}$ and 80 shots per location. For consistent performance, a 3-s warm-up time was applied for each ablation and the ablation chamber was flushed with $200 \mathrm{ml} / \mathrm{min}$ of He gas. Laser warm-up was performed only at the beginning of an array. The warm-up duration was chosen to provide similar conditions for each position of the well rather than full power as the ablation on each position was shorter than the time required by the laser to reach energy-stable operation on the flash lamp-pumped laser.

After production of the wells, the slides were transferred to a vial with ultrapure $\mathrm{H}_{2} \mathrm{O}$ (Milli-Q, $18.2 \mathrm{M} \Omega \mathrm{cm}$ ) and ultrasonicated for $15 \mathrm{~min}$ to remove debris from the surface.

Afterwards, the slide was removed from the vial and excess water eliminated with pressurized air, before transferring the slides to a laminar flow hood for the loading of the sample.

For loading, $10 \mu \mathrm{l}$ of liquid CRM (concentration range $\mathrm{ng} / \mathrm{ml}-\mu \mathrm{g} / \mathrm{ml}$ ) was positioned near a well-array and subsequently pushed over the full length of the array, using a clean slide of a small PC slide. To guarantee homogeneous loading, the diameter of the droplet should be approx. 30\% larger than the dimensions of the well-array. As only a fraction of the originally deposited droplet is used up to fill the $\mu \mathrm{DD}$ wells, excess liquid must be wiped off the slide or blown away with pressurized air.

The small amount of liquid within the wells evaporates within seconds; therefore, the $\mu \mathrm{DD}$ array is ready to use immediately after the loading step without the need for additional drying time. A shortened schematic overview of the complete procedure can be seen in Fig. 2.

\section{Sample preparation for LA-ICP-MS analysis}

Prior to sampling, the source material is thoroughly homogenized via shaking and stirring. A small amount of particles (approx. $1 \mathrm{mg}$ ) is then transferred to a vial containing $1 \mathrm{ml}$ ethanol $(99 \%$, p.a.). The suspension is then thoroughly mixed by vortexing or ultrasonication, keeping in mind that vortexing does little to destroy agglomerates and ultrasonication may result in loss of surface modifications due to cavitation. The high difference in density results in particles quickly accumulating towards the bottom of the suspension. Therefore, a strict guideline for sampling is mandatory. After $30 \mathrm{~s}$ of vortexing, the suspension is ultrasonicated for additional $30 \mathrm{~s}$. A subsample of $200 \mu \mathrm{l}$ is taken from approx. $1 \mathrm{~cm}$ above the bottom of the suspension within 
Fig. 1 Electron microscopic images. a SEM image of $\mathrm{TiO}_{2}$ clusters. b TEM micrograph of $\mathrm{TiO}_{2}$ clusters. c STEM-HAADF image of the finished catalyst material with deposited Au NP. The samples were imaged after the pretreatment process of the catalyst (oxidative treatment in $20 \% \mathrm{O}_{2}$ at $400{ }^{\circ} \mathrm{C}$ followed by reductive treatment in $5 \% \mathrm{H}_{2}$ at $300{ }^{\circ} \mathrm{C}$ in a continuous-flow fixed-bed quartz reactor under atmospheric pressure) on Lacey carbon grids

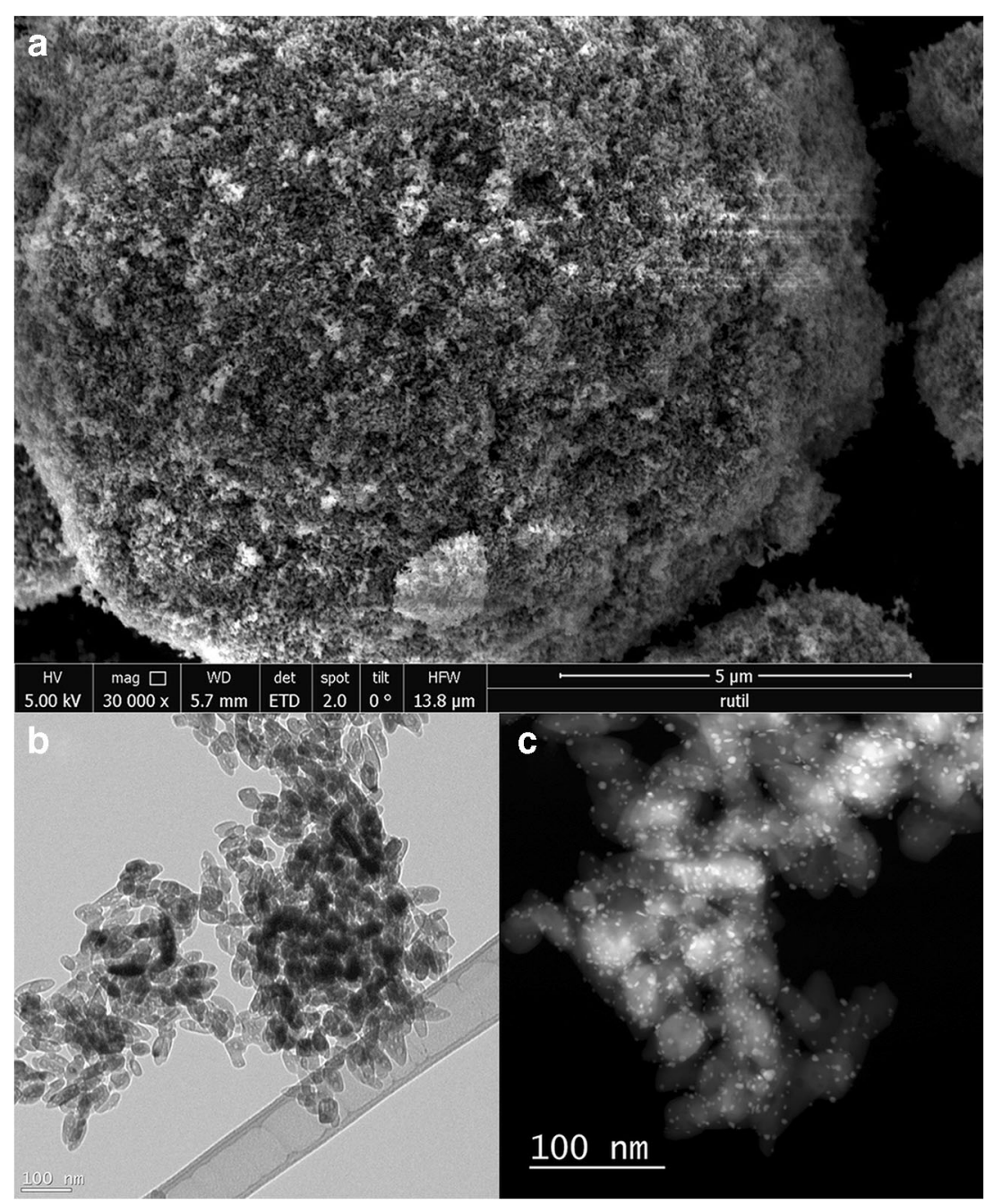

1 min of ultrasonication and transferred on a Makrolon substrate slide. Different particle suspensions varying in compositions or concentrations were deposited on the same substrate slide when there was enough space.

Effects influencing the size variation over the dimensions of the dried solution, such as the coffee ring effect, can be reduced by speeding up the evaporation of the ethanol by heating the slide to $70^{\circ} \mathrm{C}$. Nevertheless, during evaporation of the dispersing agent, agglomeration of the nanoparticles was observed, resulting in the formation of NP clusters. LA-ICP-MS analysis of the produced NP samples can be performed without any further treatment steps; in particular the particles do not require additional fixation for measurement.

As the resolution of the optical observation system in most laser ablation instruments is not capable to reliably identify single particles of less than $1-\mu \mathrm{m}$ diameter, this method is optimized for the analysis of small agglomerates of NP. However, by adjusting the particle load, single-particle analysis can also be reliably achieved for larger particles. For the evaluation of this method, we selected the commercially available reference material AUROlite $^{\mathrm{TM}}$ (Strem Chemicals).

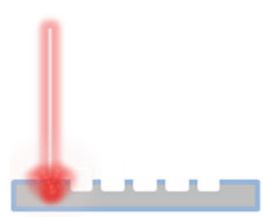

well production

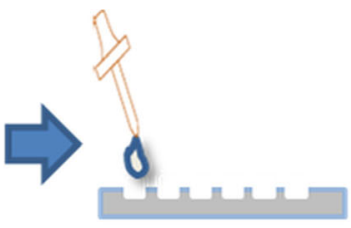

CRM deposition

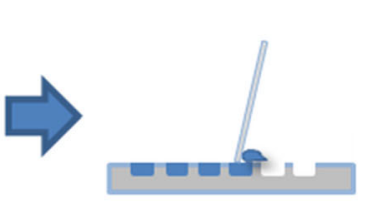

remove excess

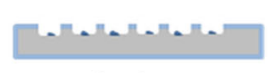

drying

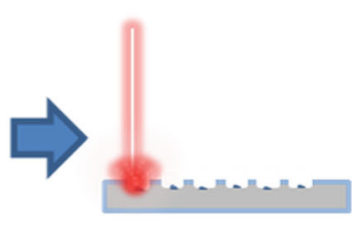

measurement

Fig. 2 Schematic $\mu$ DD production from production of the individual wells with a pulsed laser followed by analyte deposition and filling of the wells and ending in analysis via laser ablation 


\section{Measurement conditions}

The instrument settings were selected for best signal to noise $(\mathrm{S} / \mathrm{N})$ ratio and fast response to ensure sufficient data points on short transient signals. In contrast to bulk analysis, the measurement of a finite small amount such as small NP clusters does not allow for continuous stable conditions. Instead, a high and short transient signal is observed. Therefore, a compromise between high signal intensity and sufficient observation points was achieved with the conditions outlined in Table 1 . For both $\mu \mathrm{DD}$ and NP cluster analysis, the same ablation conditions were applied. Using a larger diameter in comparison to the dimensions of the $\mu \mathrm{DD}$ well, it was possible to ensure complete consumption of the deposited material within only a few laser pulses and thus a very fast washout, similar to the signal response of a particle analysis. Using the same diameter for particle analysis ensures equal amounts of substrate introduced into the plasma. As most of the material which is introduced into the plasma, thus governing the plasma conditions, is the sample substrate for both the $\mu \mathrm{DD}$ well and the nanoparticle measurement, the $\mu \mathrm{DD}$ calibration approach can be classified as "pseudo-matrix-matched".

\section{Data treatment}

The analysis of a short transient signal, such as produced by ablation of a small finite amount of material, is always a tradeoff between sensitivity and precision. Fast washout cells have been reported to significantly improve the overall sensitivity for LA-ICP-MS measurements. However, in peak-hopping analysers such as quadrupole or scanning sector field mass analysers, a short transient signal limits the amount of elements which can be observed during each measurement. Therefore, a consensus between overall signal intensity and

Table 1 Instrument settings for the LA-ICPMS analysis of $\mu \mathrm{DD}$ and $\mathrm{TiO}_{2} \mathrm{NP}$ clusters

\begin{tabular}{ll}
\hline $\begin{array}{l}\text { ICP-MS parameters } \\
\text { Plasma power }\end{array}$ & $1400 \mathrm{~W}$ \\
Nebulizer flow (Ar) & $0.9 \mathrm{l} / \mathrm{min}$ \\
Carrier flow (He) & $0.85 \mathrm{l} / \mathrm{min}$ \\
Auxiliary gas & $0.8 \mathrm{1} / \mathrm{min}$ \\
Cool gas & $14 \mathrm{l} / \mathrm{min}$ \\
Dwell time & $7 \mathrm{~ms}$ \\
Observed isotopes & ${ }^{49} \mathrm{Ti},{ }^{197} \mathrm{Au}$ \\
LA parameters & \\
Frequency & $20 \mathrm{~Hz}$ \\
Laser diameter & $80 \mu \mathrm{m}$ \\
Laser fluence & $6 \mathrm{~J} / \mathrm{cm}^{2}$ \\
Laser warm-up & $8 \mathrm{~s}$ \\
Shots per location & 40 \\
Ablation mode & $\mathrm{Spot}$ ablation \\
\hline
\end{tabular}

duration has to be reached. Preliminary experiments revealed optimum results using a dwell time of $7 \mathrm{~ms}$ and a limitation to two isotopes. In this work, a threshold of 25 cycles with more than 24 events per analysis cycle for each observed element is applied. Measurements with lower count rates or cycle count were discarded for the evaluation of the composition for each material. The measurements were then evaluated based on a robust estimation approach as presented by Walsh et al. [27]. For quantitative measurements, these requirements are expanded to the absence of spikes in the transient signal. Whilst some effort was made in the LA-ICP-MS community for both detection and elimination of spikes in transient signals, complete rejection of the measurement is the only way to ensure overall data integrity. The mean of the first 50 cycles of each measurement was used for blank correction as the substrate does not introduce any background signal for the observed $\mathrm{m} / \mathrm{z}$.

\section{Reference measurement for verification of results}

Whilst there are a few agreed-upon guidelines for the analysis of $\mathrm{TiO}_{2}$ nanoparticles (i.e., The American Society for Testing and Materials guidelines ASTM D476 - 15 and ASTM D1394 - 76), these are typically for off-the-shelf applications where only information about the size, quantity or water content of $\mathrm{TiO}_{2}$ is required. Especially for non-off-the-shelf compositions, where information about varying (surface) modifications is needed, the amount of reference guidelines is very limited as various precious metals require different digestion approaches. The chemical and thermodynamical stability of nanoparticulate $\mathrm{TiO}_{2}$, whilst making the material so highly sought after, is a challenging matrix for conventional analysis methods. In this work, two conventional liquid ICP-OES analysis methods were applied.

\section{Slurry analysis}

In slurry procedures, particulate samples are not digested with mineral acids but dispersed in suitable liquids. This method is especially useful for nanoparticulate samples which are difficult to dissolve and remain suspended long enough for the measurement. A major disadvantage of this method is the possibility of particle agglomeration as well as sedimentation of particles during the measurement, which cannot be easily corrected by addition of an internal standard to the solution.

For the slurry measurement, $15 \mathrm{mg}$ of AUROlite ${ }^{\mathrm{TM}}$ was dispersed in $10 \mathrm{ml} 2 \%$ ( $\mathrm{vol} / \mathrm{vol}$ ) $\mathrm{HNO}_{3}$ and ultrasonicated for $15 \mathrm{~min}$. From this dispersion, subsamples of $250 \mu \mathrm{l}$ and $2000 \mu \mathrm{l}$ were further diluted to $15 \mathrm{ml}$ in $2 \% \mathrm{HNO}_{3}$ with Europium as the internal standard two times each. Each sample was vortexed for $2 \mathrm{~min}$ prior to the ICP-OES analysis. To minimize the risk of clogging, a high-solid nebulizer was used in conjunction with a quartz spray chamber. The complete list 
of measurement conditions applied in the ICP-OES measurements is shown in Supplementary Table 1.

\section{Microwave digestion}

For each replicate, a subsample of 15-mg particles underwent a two-step microwave digestion. For the first digestion, a mixture of $4 \mathrm{ml} \mathrm{HNO}_{3}$ (conc.), $1 \mathrm{ml} \mathrm{H}_{2} \mathrm{O}_{2}$ (conc.) and $1 \mathrm{ml} \mathrm{HF}$ (conc.) was added to the particles and microwave-digested for $30 \mathrm{~min}$ at a maximum temperature of $180^{\circ} \mathrm{C}$ and a maximum pressure of 20 bar. Afterwards, the samples were cooled to room temperature and prepared for an additional digestion run. In the second digestion setup, $2 \mathrm{ml} \mathrm{HCl}$ (conc.) and $0.66 \mathrm{ml} \mathrm{HNO}_{3}$ (conc.) were added to the vials and digested for further $30 \mathrm{~min}$ using the same conditions.

The derived clear sample solutions are then diluted by a factor of 1000 with $2 \% \mathrm{HNO}_{3}$ (vol/vol) for subsequent ICPOES analysis. The conditions for the analysis were the same as those for the slurry measurement (see Supplementary Table 1).

\section{Results and discussion}

\section{Verification of total amounts in a $\mu D D$ well}

Before using the $\mu \mathrm{DD}$ as the calibration standard for subsequent analysis, the total amount retained in the micro wells must be determined. Profilometric means of determining the volume of each well are biased by the roundness of the stylus and therefore possibly not sufficient for calculating the amount of analyte deposited per well (see Supplementary Figure 1) and visual methods are hindered by the low contrast of the transparent Makrolon. Therefore, the total amount per well was determined by an analysis scheme based on overnight leaching of analyte-filled $\mu \mathrm{DD}$ arrays (see Supplementary Figure 2). For this, a fixed amount of wells (50) was loaded with solutions containing varying analyte concentrations $(25,50,100$ and $200 \mu \mathrm{g} / \mathrm{ml}$ in $2 \% \mathrm{HCl})$. Blanks were prepared by the application of only diluted acid.

The areas around the wells were then cut from the sheet and discarded, whereas the part containing the wells was transferred into a centrifugation vial with $1 \mathrm{ml} 2 \% \mathrm{HCl}$ (vol/vol) and ultrasonicated for $15 \mathrm{~min}$. The wells were left to leach overnight at room temperature and vortexed briefly before measuring. The concentration of the leached amount was determined by means of liquid mode ICPMS with In as the internal standard (see Supplementary Table 2 for the instrumental settings). Between the analyte contents of the applied liquid reference materials and the measured concentrations of the leachates, an excellent linear correlation was observed, enabling the calculation of the deposited mass of analyte per well. The typical error of these measurements was around 5\% RSD.
The correlation between analyte concentration and total amount per well was verified for multiple elements, which all showed a linear behaviour and followed the same slope (see Supplementary Figure 3 for the leaching calibration plot). Figure 3 shows a normalized calibration plot for $\mathrm{Au}$ and $\mathrm{Ti}$, of which a version with more elements is presented in Supplementary Figure 4. These findings indicate that the amount of deposited material is governed by the well dimensions and analyte concentration in the original droplet and is not element specific.

\section{LA-ICP-MS analysis of $\mu \mathrm{DD}$ well standards}

For this measurement approach, it is crucial to verify the absence of deposits from the original droplet outside of the $\mu \mathrm{DD}$ array to provide valid results. This was verified by performing LA-ICP-MS line scans over the surface of the slide in the original path of the droplet. As expected, the $\mu \mathrm{DD}$ wells showed huge signals for the elements of interest; however, outside the wells, some residual deposits have been found at surface defects like scratches and dust particles, as well as the rim of the slide where the droplet was pushed over after the loading. To eliminate these unintentional contributions, loading of the slides and all further treatment steps were performed in a laminar flow hood to reduce the risk of particle depositions from the lab atmosphere on the surface. Additionally, the edges of the slides were wiped clean with saturated alcohol wipes prior to LA-ICP-MS analysis. These two rather simple measures provided sufficient "decontamination" for these analytes, as no signal was measured outside of the wells in all subsequent experiments. As can be observed in Fig. 4, which shows a line scan over 5 wells in a $\mu \mathrm{DD}$ array, no signal originating from the analyte can be detected aside from the $\mu \mathrm{DD}$. The peak width of derived LA-ICP-MS signals is determined by the speed of the stage movement and the washout performance of the laser ablation system.

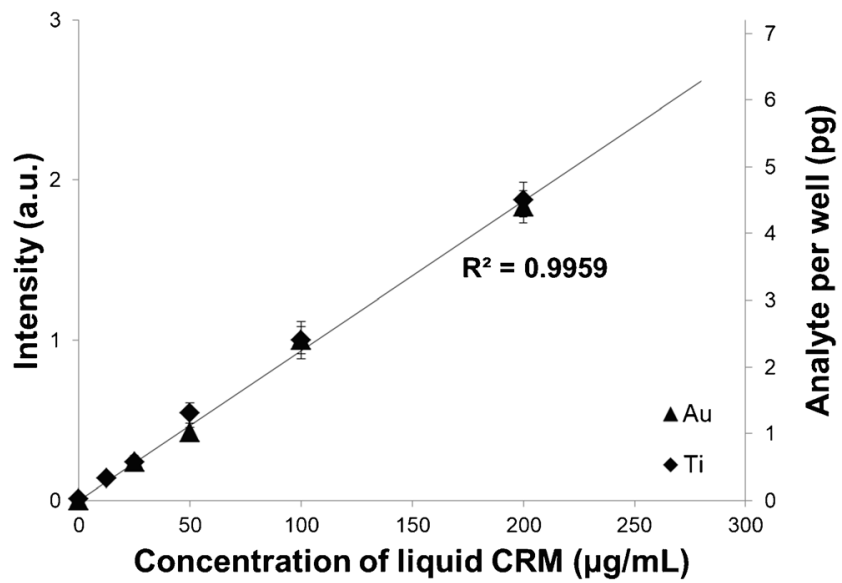

Fig. 3 Normalized calibration plot for Au and Ti $(n=20,2 \sigma)$. Quantitative amounts are based on leaching experiments 
Fig. 4 Line scan over multiple (5) $\mu \mathrm{DD}$ wells with $200 \mathrm{mg} / \mathrm{g}$ analyte, indicating no material deposition between wells

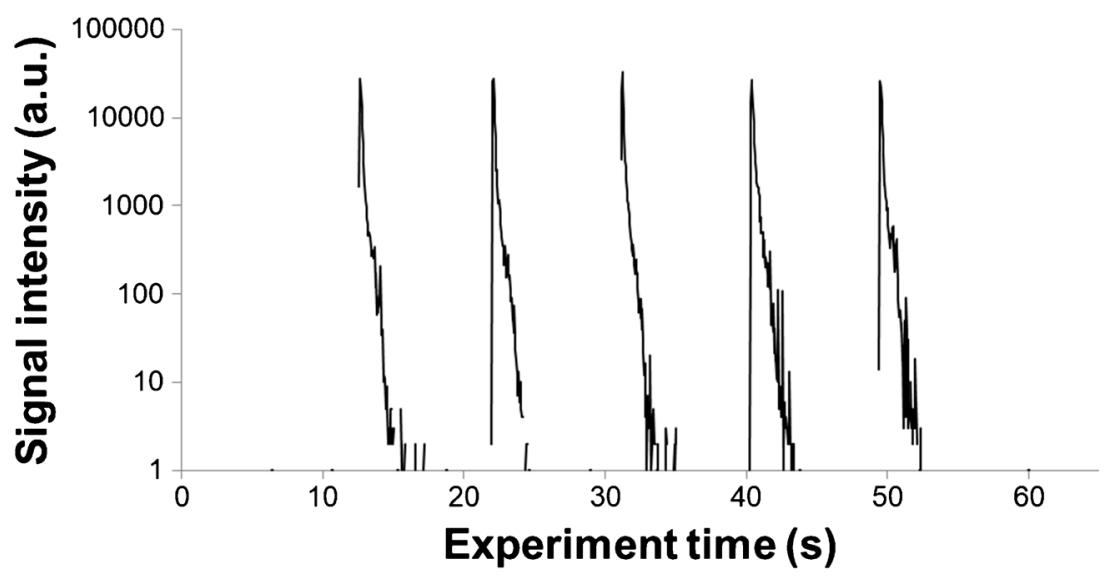

The linear range of the calibration depends on availability and solubility of the desired analytes, but typically covers 4-6 orders of magnitude. Due to the choice of substrate and standards, the limits of detection for the method are in the range of attograms to femtograms, which depend mostly on the selected criteria for data evaluation, as outlined above. The minimum amount of signal necessary to be considered was already higher than 10 times the SD of the blank; therefore, the LOD/ LOQ values were determined via the regression of the slope as this yielded a more conservative estimate. The limit of detection, derived this way, was $1.0 \mathrm{fg}$ for $\mathrm{Ti}$ and $1.3 \mathrm{fg}$ for $\mathrm{Au}$.

\section{Analysis of AUROlite ${ }^{T M}$ reference material}

For the LA-ICP-MS analysis, a suspension of AUROlite ${ }^{\mathrm{TM}}$ in ethanol was dispersed on Makrolon ${ }^{\circledR}$ and transferred to the laser chamber. For the selection of clusters to be analysed, it is important to only select clusters which are isolated enough from others to be ablated with the same beam diameter as the $\mu \mathrm{DD}$, to establish similar measurement conditions. Therefore, only clusters which were barely visible in the optical microscope of the laser and well separated from other visible clusters were analysed. When comparing the transient signals and the overall intensities of the respective signals for $\mu \mathrm{DD}$ and AUROlite ${ }^{\mathrm{TM}} \mathrm{NP}$ clusters (see Fig. 5), an overall good agreement in the signal shape of the standard material and sample can be observed. The $\mathrm{Ti}$ and $\mathrm{Au}$ signals observed during laser ablation of individual NP clusters were converted into absolute amounts using the determined calibration functions. This data evaluation approach leads to only one single data point for each NP cluster, since replicate measurements of the same cluster were not possible. In total, a number of 20 NP clusters were analysed, and 17 of these clusters were considered for evaluation of which the smallest amount exceeded the limit of quantification for $\mathrm{Ti}(3.0 \mathrm{fg})$ and $\mathrm{Au}$ (3.9 fg) by approx. a factor 50 .

The reference value for $\% \mathrm{wt} \mathrm{Au}$ in AUROlite ${ }^{\mathrm{TM}}$ particles is $0.8 \%$ wt, with no stated uncertainty, whilst the microwave- assisted digestion and LA-ICP-MS measurement succeeded in reaching the same conclusion. With $0.77 \% \mathrm{wt} \pm 0.05 \% \mathrm{wt}$ for the digestion and $0.74 \% \mathrm{wt} \pm 0.13 \%$ wt for the LA-ICPMS measurement, respectively, both equally overlap with the certified value. The $\mu$ DD method shows good agreement to the microwave digestion with a moderate decrease in precision. This outcome demonstrates the applicability of the developed LA-ICP-MS procedure. The slurry method resulted in a significant over-estimation at $1.5 \% \mathrm{wt} \pm 0.2 \% \mathrm{wt}$ and did not agree with the certified value. This can be explained by erosion of the surface modification due to the acidic solution or due to cavitation during the ultrasonication step. Furthermore, the slurry method, whilst seemingly ideal as it does not require digestion of the sample, struggled with sedimentation and especially agglomeration of the samples leading to unpredictable intensities during measurements as well as frequent clogging of the nebulizer.

Compared to the two reference methods for assessment of the gold content, which provide only bulk information, the LA-ICP-MS procedure also hints about the material homogeneity. As can be deduced from Fig. 6, which presents the

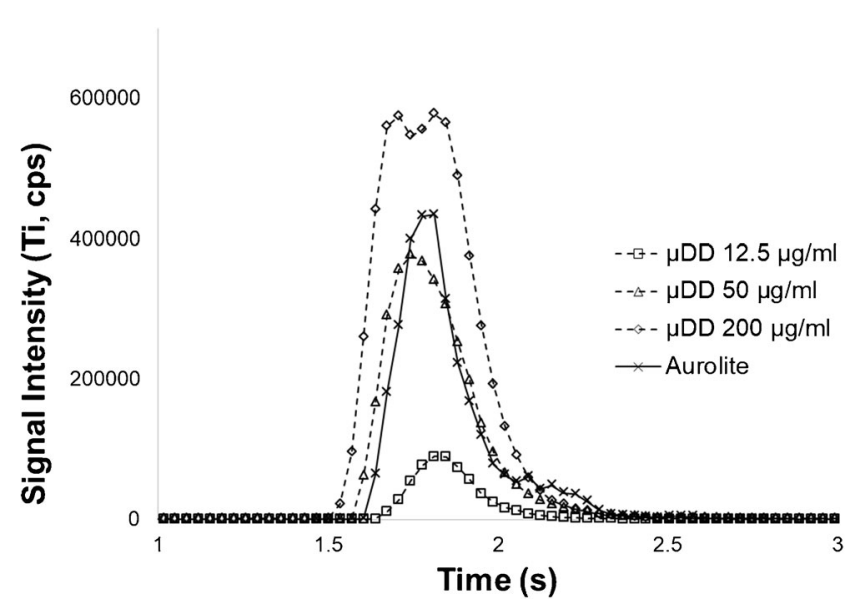

Fig. 5 Comparison of transient signals from $\mu \mathrm{DD}$ and NP clusters; timescale modified for presentation purposes. The transit line was modified to broaden the transient signal to provide more data points per analysis run 
dependence of the determined $\mathrm{Au} / \mathrm{Ti}$ ratio from the analysed sample mass, the composition of the AUROlite ${ }^{\mathrm{TM}}$ reference material is inhomogeneous. Results observed for the investigated NP clusters provide no information about measurement uncertainty due to the integration of whole peak intensities and complete consumption of the material in one measurement, resulting in only one single data point per LA-ICPMS analysis. When larger clusters and thus higher amounts of AUROlite ${ }^{\mathrm{TM}}$ were ablated, an excellent agreement with the certified composition was found. However, for experiments with lower sample intakes, a distinct deviation of the achieved $\mathrm{Au} / \mathrm{Ti}$ ratios from the target value was observed. Problems in the quality of LA-ICP-MS analysis could be excluded as a possible reason for this outcome, since the $\mathrm{Au}$ and Ti signals recorded in these measurements were still orders of magnitude above the respective limits of quantification and the analysis of reference $\mu \mathrm{DD}$ with similar amounts does not suffer from similar deviation as can be seen in Supplementary Figure 5 (approx. 5\% RSD). Hence, the deviation from the certified value indicates that the homogeneity of the AUROlite ${ }^{\mathrm{TM}}$ material is limited when looking towards sampling sizes of less than 300 pg per measurement (which corresponds to amounts of less than $3 \mathrm{pg} \mathrm{Au}$ ).

\section{Analysis of custom-designed surface-modified $\mathrm{TiO}_{2} \mathrm{NP}$}

Reaction rates and turnover frequencies (TOFs) are essential characteristics of a catalyst. For calculating reaction rates and relating them to the amount of catalytically active metal nanoparticles, exact knowledge of the respective metal loading of the catalyst materials is essential. However, due to the application of different synthesis routes and sometimes occurring variations in the quality of synthesis, information about the exact loading is often not known. In this work, three Au catalysts on different NP support materials (anatase, rutile and an $80 / 20$ blend of anatase and rutile) were synthesized in the same way and then analysed to evaluate their respective $\mathrm{Au}$ loading. In contrast to the previously described analysis of the AUROlite ${ }^{\mathrm{TM}}$ reference material, no significant dependence of the determined composition from the analysed sample size was found. A comparison of 20 individual clusters for each composition is available in Supplementary Figure 6. Whilst the rutile and anatase substrate both deposited similar amounts of $\mathrm{Au}$ (rutile $2.9 \% \mathrm{wt} \pm 0.12 \% \mathrm{wt}$, anatase $2.8 \% \mathrm{wt} \pm 0.17 \% \mathrm{wt}$ ), the $80 / 20$ blend incorporated almost $0.5 \%$ more $\mathrm{Au}$ onto its surface $(3.4 \% \mathrm{wt} \pm 0.24 \% \mathrm{wt})$. Figure 7 depicts the reaction rate per gram Au loading, as determined above, for the gas phase oxidation of ethanol to acetaldehyde in a fixed bed flow reactor. The exact quantification of $\mathrm{Au}$ on the catalyst surface allows the clear conclusion that $\mathrm{Au} /$ rutile is the most active support and a mixture of anatase and rutile is less beneficial than anatase. Despite the higher Au loading of the 80/20

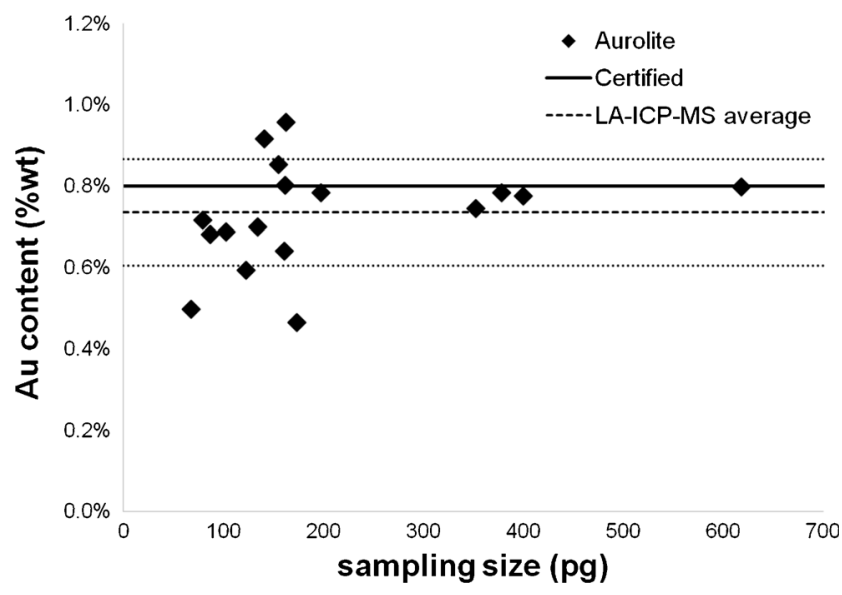

Fig. 6 The determined $\mathrm{Au}$ content of the AUROlite material in correlation to the sampling size. When analysing less than $200 \mathrm{pg}$, significant variation in the determined $\mathrm{Au}$ content can be observed

blended support, this catalyst shows lower activity, which might attributed to the heterogeneity of the Au dispersion.

\section{Conclusions and outlook}

In this work, it was demonstrated that the combination of liquid CRM with self-aliquoting micro-wells can be used for quantification in LA-ICP-MS with sample sizes in the picogram range. The absolute amount of analyte required for quantitative analysis is about $3.0 \mathrm{fg}$ for $\mathrm{Ti}$ and $3.9 \mathrm{fg}$ for $\mathrm{Au}$ and thus significantly reduced when compared to wet chemical approaches used for conventional bulk measurements. Thus, sampling of microgram subsamples during synthesis can be easily used for at-line quality control of a NP synthesis, as the samples require little handling aside from dispersion in alcohol and subsequent pipetting on a suitable substrate. This advantage is of special importance for analysis of valuable

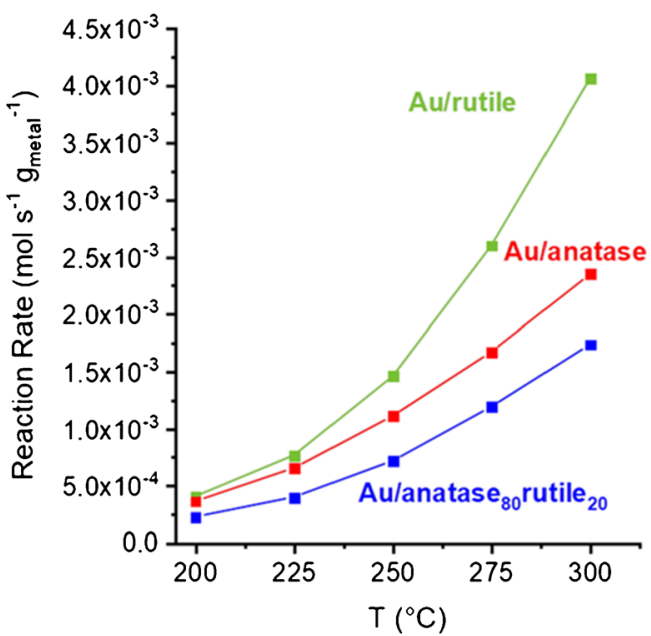

Fig. 7 Reaction rate of the individual catalyst materials normalized to the determined $\mathrm{Au}$ content, in regard to reaction temperature 
samples such as the investigated catalyst material containing expensive noble metals.

Applicability of the proposed procedure has been demonstrated by analysis of AUROlite ${ }^{\mathrm{TM}}$ reference material, indicating that the homogeneity of the material is limited for very small sampling amounts and the certified value is only achieved when more than approximately $300 \mathrm{pg}$ is used for analysis. This observation agrees with TEM observations made prior to this work [28]. For smaller amounts, increased differences to the target value were observed, which did not originate from errors in the LA-ICP-MS analysis. Thus, the presented LA-ICP-MS provides additional information about the sample homogeneity which is not accessible with conventional digestion or slurry procedures. However, the precision of LA-ICP-MS analysis of a subset of picogram to nanogram clusters cannot (yet) compete with bulk measurement procedures.

The ease of $\mu \mathrm{DD}$ production and high flexibility in terms of composition have to be considered as the major advantage of this procedure, as it allows simple adjustment to the needs of the actual task, a prerequisite for the characterization of novel composite materials.

Another major benefit of the developed approach is the possibility of sample storage. Individual $\mu \mathrm{DD}$ reference standards have shown no significant amount of deterioration over the timeframe of multiple months and batch-to-batch variation can be minimized by well-defined operation procedures. Long-term stability can be further improved by covering the substrate surface with a thin layer of polyimide or similar polymers; thus, the possibility of sample contamination or the introduction of interferences is reduced.

Acknowledgements We thank M. Palir and K. Dobrezberger for the SEM imaging of the rutile support.

Funding Open access funding provided by TU Wien (TUW). This project was financially supported by the Austrian Science Fund (FWF) by project [I2158-N28] and the IAEA joint research project PO_84_ 201503114_0_US and PO_84_201801638_0_US.

\section{Compliance with ethical standards}

Conflict of interest The authors declare that they have no conflict of interest.

Open Access This article is licensed under a Creative Commons Attribution 4.0 International License, which permits use, sharing, adaptation, distribution and reproduction in any medium or format, as long as you give appropriate credit to the original author(s) and the source, provide a link to the Creative Commons licence, and indicate if changes were made. The images or other third party material in this article are included in the article's Creative Commons licence, unless indicated otherwise in a credit line to the material. If material is not included in the article's Creative Commons licence and your intended use is not permitted by statutory regulation or exceeds the permitted use, you will need to obtain permission directly from the copyright holder. To view a copy of this licence, visit http://creativecommons.org/licenses/by/4.0/.

\section{References}

1. Fujishima A, Rao TN, Tryk DA (2000) Titanium dioxide photocatalysis. J Photochem Photobiol C: Photochem Rev 1:121. https://doi.org/10.1016/s1389-5567(00)00002-2

2. Ndolomingo MJ, Bingwa N, Meijboom R (2020) Review of supported metal nanoparticles: synthesis methodologies, advantages and application as catalysts. J Mater Sci 55:6195-6241. https:// doi.org/10.1007/s10853-020-04415-x

3. Haruta M (1989) Gold catalysts prepared by coprecipitation for low-temperature oxidation of hydrogen and of carbon monoxide. J Catal 115:301-309. https://doi.org/10.1016/0021-9517(89) 90034-1

4. Haruta M, Kobayashi T, Sano H, Yamada N (1987) Novel gold catalysts for the oxidation of carbon monoxide at a temperature far below $0{ }^{\circ} \mathrm{C}$. Chem Lett 16:405-408. https://doi.org/10.1246/cl. 1987.405

5. Ebdon L, Foulkes M, Sutton K (1997) Slurry nebulization in plasmas. J Anal At Spectrom 12:213-229. https://doi.org/10. $1039 / a 604914 a$

6. Jarvis KE, Williams J (1989) The analysis of geological samples by slurry nebulisation inductively coupled plasma - mass spectrometry (ICP-MS). Chem Geol 77:53-63. https://doi.org/10.1016/00092541(89)90015-6

7. Williams JG, Gray AL, Norman P, Ebdon L (1987) Feasibility of solid sample introduction by slurry nebulisation for inductively coupled plasma mass spectrometry. J Anal At Spectrom 2:469. https://doi.org/10.1039/ja9870200469

8. Abu-Samra A, Morris JS, Koirtyohann SR (1975) Wet ashing of some biological samples in a microwave oven. Anal Chem 47: 1475-1477. https://doi.org/10.1021/ac60358a013

9. Mudunkotuwa IA, Renée Anthony T, Grassian VH, Peters TM (2016) Accurate quantification of tio $_{2}$ nanoparticles collected on air filters using a microwave-assisted acid digestion method. J Occup Environ Hyg 13(1):30-39. https://doi.org/10.1080/ 15459624.2015.1072278

10. Pisonero J, Fernández B, Günther D (2009) Critical revision of GDMS, LA-ICP-MS and SIMS as inorganic mass spectrometric techniques for direct solid analysis. J Anal At Spectrom 24(9):11451160. https://doi.org/10.1039/B904698D

11. Kappel S, Boulyga SF, Prohaska T (2012) Direct uranium isotope ratio analysis of single micrometer-sized glass particles. J Environ Radioact 113:8-15. https://doi.org/10.1016/j.jenvrad.2012.03.017

12. Pointurier F, Pottin A-C, Hubert A (2011) Application of nanosecond-UV laser ablation-inductively coupled plasma mass spectrometry for the isotopic analysis of single submicrometersize uranium particles. Anal Chem 83:7841-7848. https://doi.org/ $10.1021 /$ ac201596t

13. Benešová I, Dlabková K, Zelenák F, Vaculovič T, Kanický V, Preisler J (2016) Direct analysis of gold nanoparticles from dried droplets using substrate-assisted laser desorption single particleICPMS. Anal Chem 88:2576-2582. https://doi.org/10.1021/acs. analchem.5b02421

14. Wang M, Zheng L-N, Wang B, Chen HQ, Zhao YL, Chai ZF, Reid HJ, Sharp BL, Feng WY (2014) Quantitative analysis of gold nanoparticles in single cells by laser ablation inductively coupled plasma-mass spectrometry. Anal Chem 86:10252-10256. https:// doi.org/10.1021/ac502438n

15. Fittschen UEA, Bings NH, Hauschild S, Förster S, Kiera AF, Karavani E, Frömsdorf A, Thiele J, Falkenberg G (2008) Characteristics of picoliter droplet dried residues as standards for direct analysis techniques. Anal Chem 80:1967-1977. https://doi. org $/ 10.1021 /$ ac $702005 x$

16. Rovelli S, Nischkauer W, Cavallo DM, Limbeck A (2018) Multielement analysis of size-segregated fine and ultrafine particulate via 
laser ablation-inductively coupled plasma-mass spectrometry. Anal Chim Acta 1043:11-19. https://doi.org/10.1016/j.aca.2018.10.026

17. Villaseñor Á, Greatti C, Boccongelli M, Todolí JL (2017) A dried droplet calibration approach for the analysis of solid samples through laser ablation - inductively coupled plasma mass spectrometry. J Anal At Spectrom 32:587-596. https://doi.org/10.1039/ c6ja00343e

18. Deegan RD, Bakajin O, Dupont TF, Huber G, Nagel SR, Witten TA (1997) Capillary flow as the cause of ring stains from dried liquid drops. Nature 389:827-829. https://doi.org/10.1038/39827

19. Deegan RD, Bakajin O, Dupont TF, Huber G, Nagel SR, Witten TA (2000) Contact line deposits in an evaporating drop. Phys Rev E 62:756-765. https://doi.org/10.1103/physreve.62.756

20. Nischkauer W, Vanhaecke F, Bernacchi S, Herwig C, Limbeck A (2014) Radial line-scans as representative sampling strategy in dried-droplet laser ablation of liquid samples deposited on pre-cut filter paper disks. Spectrochim Acta B At Spectrosc 101:123-129. https://doi.org/10.1016/j.sab.2014.07.023

21. Bellis DJ, Santamaria-Fernandez R (2010) Ink jet patterns as model samples for the development of LA-ICP-SFMS methodology for mapping of elemental distribution with reference to biological samples. J Anal At Spectrom 25:957. https://doi.org/10.1039/b926430b

22. Van Malderen S, Vergucht E, De Rijcke M et al (2016) Quantitative determination and subcellular imaging of $\mathrm{Cu}$ in single cells via laser ablation-ICP-mass spectrometry using high-density microarray gelatin standards. Analytical Chemistry 88(11):5783-5789. https:// doi.org/10.1021/acs.analchem.6b00334
23. Nischkauer W, Vanhaecke F, Limbeck A (2016) Self-aliquoting micro-grooves in combination with laser ablation-ICP-mass spectrometry for the analysis of challenging liquids: quantification of lead in whole blood. Anal Bioanal Chem 408:5671-5676. https:// doi.org/10.1007/s00216-016-9717-3

24. Weiss M, Riedl H, Moares V, Mayrhofer PH, Limbeck A (2020) Laser based analysis of transition metal boride thin films using liquid standards. Microchem J 152:104449. https://doi.org/10. 1016/j.microc.2019.104449

25. Zanella R, Giorgio S, Henry CR, Louis C (2002) Alternative methods for the preparation of gold nanoparticles supported on TiO2. J Phys Chem B 106:7634-7642. https://doi.org/10.1021/ jp0144810

26. Zanella R, Delannoy L, Louis C (2005) Mechanism of deposition of gold precursors onto $\mathrm{TiO} 2$ during the preparation by cation adsorption and deposition-precipitation with $\mathrm{NaOH}$ and urea. Appl Catal A Gen 291:62-72. https://doi.org/10.1016/j.apcata.2005.02.045

27. Walsh SJ, Dzigal N, Chinea-Cano E, Limbeck A (2017) Simple robust estimation of uranium isotope ratios in individual particles from LA-ICP-MS measurements. J Anal At Spectrom 32:11551165. https://doi.org/10.1039/c7ja00089h

28. Nagl A (2019) Selective oxidation of ethanol on modified supported $\mathrm{Au}$ catalysts: from fundamental understanding to improved performance. Dissertation, TU Wien

Publisher's note Springer Nature remains neutral with regard to jurisdictional claims in published maps and institutional affiliations. 